\title{
Plasma Physics approach for the Interaction of Electromagnetic Wave with Ionosphere
}

\author{
Abdulridha Salman Hassani ${ }^{1}$, Halla Hameed $\mathrm{Ali}^{2}$ \\ ${ }^{I}$ (College of Science / University of Baghdad Name, Iraq) \\ ${ }^{2}$ (College of Science / University of Baghdad, Iraq)
}

\begin{abstract}
Abstract : The concept of ionospheric modifications is a part of a much more common class of research today known as "Active Experiments" in auroral plasma. The interaction of High power electromagnetic Radio waves with auroral plasma is one of that field which has been have been investigated by this study. Plasma in that auroral region might be illuminated via electromagnetic waves for a prolonged period and therefore, undergo accumulative resonances and perturbations due to its slow transport rates, weak convection and long lifetime of plasma, mainly nearby the peak of profile of ionospheric electron density. A plasma can be resonance at a particular height in the ionosphere includes a equivalent electromagnetic wave frequency. These types of plasma resonances are able to improve the local fields of electromagnetic, and as a result their interactions with plasma particles causing density perturbations, turbulences, field aligned striations and local heating. These kinds of effects will be more obvious when the electromagnetic wave frequency is close to foF2, the resonance frequency nearby the peak of the profile of ionospheric electron density. Optical emissions may also be enhanced during such conditions. This work aim to investigate the interaction of EM with ionosphere layers $D, E$ and $F$. The simulation model run under COMSOL Multiphasic software. The EM selected to be at $10 \mathrm{MHz}$ and $2.4 \mathrm{GH}$, the electron density has been taken to be $\left(10^{8}, 10^{10}\right.$ and $\left.10^{11}\right)$ for $D, E, F$ ionospheric layers respectively. The simulation results have been recorded and analyzed for: electron temperature of plasma, electron density of plasma, electric potential of plasms, resistive losses, electric field norm and the relation between the input port power and the time. The results provide a clear and important information about interaction of EM with ionosphere that can help to predicate there transmission and its range for many purpose.
\end{abstract}

Keywords: Ionospheric, Electromagnetic Wave Interaction, auroral plasma.

\section{Introduction}

The ionosphere is a type of naturally sourced plasma formed from soft $\mathrm{x}$-ray radiation and solar photoionization. UV radiation via the sun can ionizes the atmospheric elements. Free thermal $(1<\mathrm{eV})$ ions and electrons are existing in the earth upper atmosphere in about the range between $50 \mathrm{~km}$ and $6000 \mathrm{~km}$. In this zone, the temperature of atmospheric is first raises with altitude to reach total maximum value $(900 \mathrm{~K})$ and after that gets to be constant with altitude (as shown in Figure 1). In the ionosphere, an equilibrium in between photoionization and several loss mechanisms gives increase to an equilibrium density of ions and free electrons which has a horizontally stratified structure. These electrons density is a function of the height over the earth surface and it is significantly impacted by the effects of sunset and sunrise, mainly at the lower altitudes. The existence of those free electrons in the ionosphere may influence the propagation of radio waves [1].

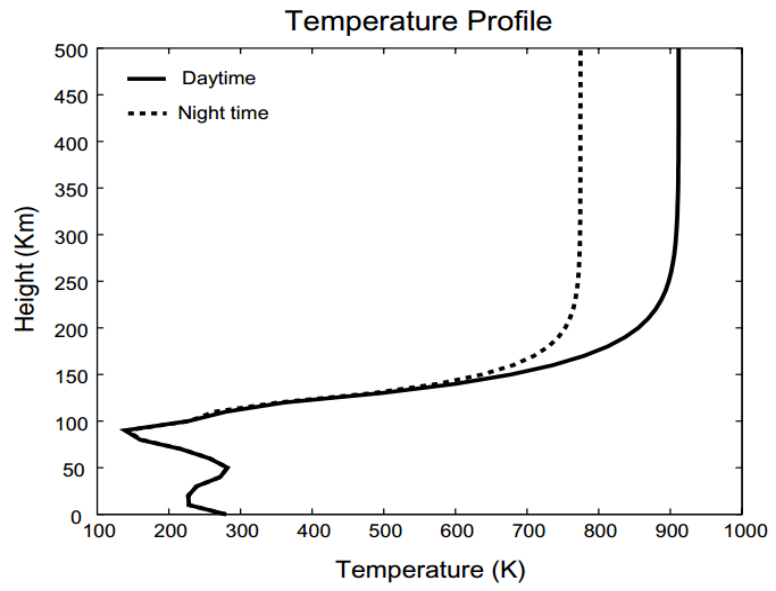

Figure 1: The earth's atmospheric temperature profiles calculated from the MSIS-E-90 Atmospheric Model at HIPAS, Alaska [1]. 
In comparison with laboratory plasmas, the ionospheric plasma is usually effectively unbounded and has long-lived. The transport rate is generally low so that a radiation effects and a cumulative turbulent can happen. Because of this, an average power of around one megawatt may excite a wide range of spatial and temporal plasma instabilities. In a $100 \mathrm{~km}$ height, the energy flux is about $0.56 \mathrm{~mW} / \mathrm{m} 2$, ignoring ionospheric absorption. However, the transmitted power is much more powerful than who utilized in the laboratory plasma experiments, this pumping energy is quite weak in comparison to the thermal energy of ionospheric plasma. The ratio is on order of $10^{4}-10^{3}$. The characteristic scale lengths of several plasma phenomena are far small compared to ionospheric plasma size, thus experiments could be conducted within a homogeneous environment [2].

\section{Literature Survey}

In 2003, D. L. Tang et.al. [3] studied the absorption, reflection, and transmission of electromagnetic waves by a nonuniform plasma slab immersed in an ambient uniform magnetic field of various strengths. They discussed the effects of the plasma parameters and magnetic field strength on the absorbed, reflected, and transmitted power. The calculation results show that the effects of the magnetic field strength and density gradient on the absorbed power, as well as the frequency band of resonant absorption, are significant. Broadband absorption of electromagnetic waves can be achieved by changing the magnetic field strength and plasma density.

In 2009, C. S. Gurel and E. Oncu [4] searched in the interaction of an electromagnetic wave and an inhomogeneous plasma slab with electron distribution in the form of partially linear and sinusoidal profiles to determine new refection, absorption and transmission characteristics. The researchers found that broadband and tunable absorption performance of the plasma layer accompanied with narrow-band refection characteristic is presented as the function of electron density profile parameters and external magnetic field excitation. They proposed from the result that plasma layer can be useful as a new absorbing layer for shielding and stealth applications. In 2011, K. Papadopoulos et.al [5] gave the first experimental demonstration of HF driven currents in the ionosphere at low ELF/ULF frequencies without relying in the presence of electrojets. The effect was predicted by theoretical/ computational means in a recent letter and given the name Ionospheric Current Drive (ICD). The effect relies on modulated F- region HF heating to generate Magneto- Sonic (MS) waves that drive Hall currents when they reach the E- region. The Hall currents inject ELF waves into the Earth- Ionosphere waveguide and helicon and Shear Alfven (SA) waves in the magnetosphere.

\section{Ionosphere Structure}

The ionosphere is traditionally put into the D-, E-, and F-regions (as shown in Figure 2). The D-region is situated between $60 \mathrm{~km}$ and $95 \mathrm{~km}$, the E-region situated between $95 \mathrm{~km}$ and $150 \mathrm{~km}$, and then the F-region is located above $150 \mathrm{~km}$. In daytime, it's possible to identify two separate layers on the F-region, the lower layers (F1) and the upper layers (F2). While in night time, both of these layers combine into a single layer. The merged effect of gravitationally minimizing densities of neutral molecules and atoms as well as increasing the intensity of ionizing solar UV radiation as increasing altitudes, provides a maximum plasma density throughout daytime within the F-region in a few hundred kilometers altitude. Through daytime, the ratio of concentration of charged particles to neutral particles may vary from $10^{8}$ at $100 \mathrm{~km}$ to $10^{4}$ at $300 \mathrm{~km}$ and 101 at $1000 \mathrm{~km}$ altitude $[6,7]$.

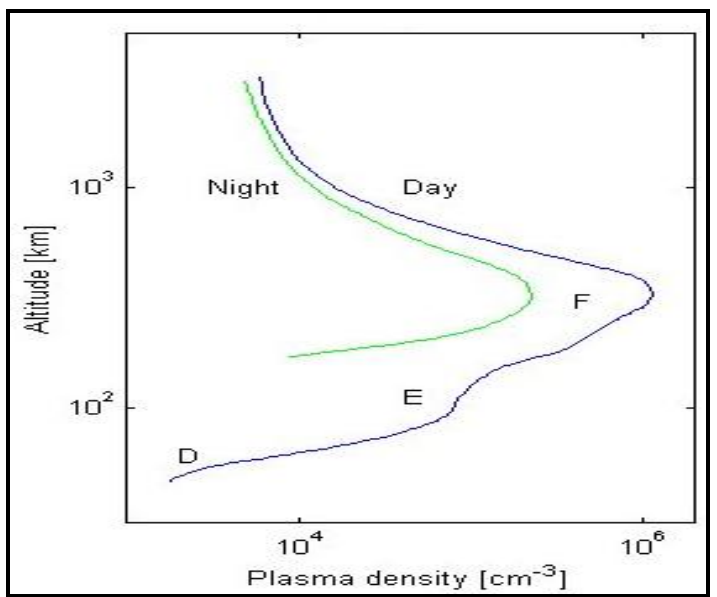

Figure 2: Several layers of the ionosphere. Actual electron density profiles range through a wide range and be dependent significantly on sun-spot number, seasons, time of day, and whether the ionosphere is disturbed or not [7]. 
The D-region is both an absorbing and refracting medium for electromagnetic radio waves. As the electromagnetic radio waves transfer over the ionosphere, it will interact with the electrons and make them oscillating. With the higher concentration of each atomic and molecular gas particles within the D-region, these kinds of oscillations are damped by collisions between the molecules of gas and electrons. Over the D-region the frequency of collision reduces significantly with height, approximately proportionate to the pressure of neutral gas, while most electron density raises quickly with height. In this zone, chemical operations are most significant, molecular ions dominate, and $\mathrm{O}, \mathrm{O}_{2}$, and $\mathrm{N}_{2}$ are the majority of considerable neutral species. The density of electron is sharply decreased at night due to the molecular ions have a greatly greater recombination rate with electrons rather than atomic ions. Therefore, the radio waves linear absorption in this zone is varies from a typical 4 to10times (or 6-10dB) through daytime to some lower rate over nighttime. The behavior of the E-region is being subject to close solar control. In this region, the basic chemical reactions are not as complicated as those in the D-region. The major ions are $\mathrm{NO}^{+}, \mathrm{O}_{2}^{+}$, and $\mathrm{N}_{2}+$. The total ion density is of the order of $10^{5} \mathrm{~cm}^{3}$, while the neutral density is greater than $10^{11} \mathrm{~cm}^{3}$. Thus, the E-region plasma is ionized weakly, and the collisions among charged particles aren't important. The casual E layer is composed mostly of fairly dense patches of electrons having a horizontal extent of many kilometers and tend to be considered to be made by meteoric particles. Besides photo-ionization, the energetic particles via the solar wind collid with neutral gas causing further plasma production in the E-region. The F-region is greatly denser compared to the E-region and also greatly ionized, in which the greatest electron density finds in the range $200-400 \mathrm{~km}$. In this area, the $\mathrm{O}^{+}$and $\mathrm{O}$ atomic species dominate. In daytime, an additional bulge is noted in the curve of electron density under the maximum. Here is the F1 layer, in which the major maximum electron density detects is the F2 layer. The F2 layer highest ionospheric electron plasma frequency could be the critical frequency "foF2". The density of peak ion within the F2-layer is nearly a factor of 10 more than that in the E-region, whilst the neutral density $\left(10^{8} \mathrm{~cm}^{3}\right)$ remains requests of value higher than the ion density. The plasma in this area is somewhat collisions and ionized over neutral particles and charged should be considered. The effective collision frequency of electron is $500 \mathrm{~Hz}$ in the altitude of $200 \mathrm{~km}$ as well as the thermal electron imply free path is approximately $1 \mathrm{~km}$. The scale level of the ambient plasma density profile means the height variances for the density to maximize by a factor of $\mathrm{e}$ from its reference value. In the F-region bottom boundary, in which the pump wave is reflected, the size height is usually $50 \mathrm{~km}$. It's larger compared to vacuum pump wavelength of $30-100 \mathrm{~m}$. Sun rising and sunset have little or no influence in the F-region. Even so, the geomagnetic field performs a significant role within the structure and dynamics with this region. As collisions of charged particles are adequately lower frequent in this area, the charged particles motion is limited by the earth magnetic field. At higher latitudes, in which the geomagnetic field is approximately perpendicular to the earth surface the area lines can expand in to the interplanetary medium. The charged particles ejected from the sun are therefore taken down towards the ionosphere and represented as the aurora. The distribution of electron within these layers is be subject to geographical variations, daily, diurnal and seasonal and also the magnetic disruption affiliates with solar activities. Among those layers, the F2 layer is the most variable. As a solar flare occurs, the rapid raise of x-ray emission can result in a big increase of ionization within the lower ionosphere regions of D-, E- and F -, within the earth sunlit side. Sufficient long lived and large solar flares can make geomagnetic storms that can lead to ionospheric disturbances. The magnetic field of earth differs partially because of the induced earth currents and the external currents. The Quiet days are the days at regular transient magnetic variations, while disturbed it is the days which are magnetically disturbed. Within these disrupted days, further currents circulate within the ionosphere. Besides the visible aurora, the process of ionization is improved, corresponding to the rise in sporadic absorption of E and D-region [1].

\section{Waves Propagation in the Ionosphere}

When a powerful electromagnetic (EM) wave is transmitted into the ionosphere from the ground, it can excite a wide range of plasma processes. Most of these processes are plasma turbulence excited by the EM wave through parametric instabilities. Parametric instabilities play the dominant role near the reflection height of the EM wave. The decay modes of the parametric instabilities at this height are Langmuir wave and ion acoustic wave/field-aligned density irregularities. However, in the high latitude region, the UH resonance layer becomes important. Perpendicularly propagating modes such as the UH wave/electron Bernstein (EB) wave and the lower-hybrid (LH) wave/ion Bernstein wave/fieldaligned density irregularity are involved in the parametric instabilities [8].

For interaction of electromagnetic waves with plasma, the electric field and power density of radio wave (RF) radiation increases continuously in a plasma, the response of the plasma to the incident energy changes discontinuously. This follows from a complex set of competing processes, each generally with its own power dependent threshold, and plasma instabilities, each with their own growth and decay rate. Non-linear power dependencies, and dependencies on boundary conditions, past histories of the plasma conditions, dependence on proximity to plasma resonances, and nonlinear mixing in the plasma leading to up or down 
conversion with respect to resonances, all conspire to make experimental guidance invaluable to theoretical development, and vice versa [9].

The RF propagation and emission environment is affected through the ULF to $\mathrm{GHz}$ range by absorption (heating), lensing, scattering, modulation, and stimulated emission. The optical background and emission is affected over a wide spectrum by electron impact and temperature enhancements, altering translational, rotational, and vibrational temperatures. At higher power densities, there is the further need to consider effects of raising the fine structure population distribution of atomic oxygen. An adequate understanding of the processes, if not a predictive capability of the consequences of sending very high power density RF into the ionosphere, represents common ground for many scientific discipline, agency, national and international, civilian and defense goals and missions.

A parameter of particular importance for the interaction between electromagnetic fields and plasma is the plasma frequency $\omega_{\mathrm{p}}$. The plasma frequency is the resonance frequency for collective oscillations of the electrons about their equilibrium positions [9]:

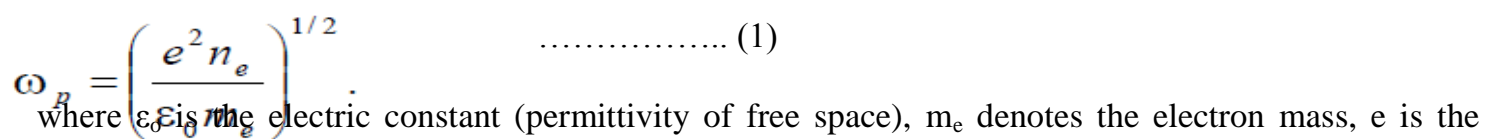
elementary charge and $n_{\mathrm{e}}$ the electron density. The plasma frequency can be seen as the inverse of the typical time for collective action, just as the Debye length is the typical length $\lambda_{D}[9]$ :

$$
\lambda_{D}=\left(\frac{\varepsilon_{0} k_{B} T_{e}}{e^{2} n_{e}}\right)^{1 / 2}
$$

where $\mathrm{k}_{\mathrm{B}}$ is the Boltzmann constant and $\mathrm{T}_{\mathrm{e}}$ is the electron temperature. The Debye length denotes a distance beyond which the potential of an individual charge is shielded by surrounding mobile charges. That implies, that on spatial scales shorter than $\lambda_{\mathrm{D}}$ the presence and effects of individual charges are evident. On a scale larger than $\lambda_{\mathrm{D}}$, charged particle interactions tend to occur through collective motions, such as electron- and ion-acoustic waves. One can also use this concept to separate the behavior of the charges into collisional (spatial scale $<<\lambda_{\mathrm{D}}$, binary collisions dominate the interaction) and collective (spatial scale $>>\lambda_{\mathrm{D}}$, plasma is quasineutral, collective behavior dominates).

An electromagnetic wave with frequency $\omega_{\mathrm{L}} \gg \omega_{\mathrm{p}}$ can penetrate into the plasma and interacts with a medium of resonance frequency $\omega_{\mathrm{p}}$. The electrons within the plasma can not follow the rapid oscillation and the wave propagates. The electromagnetic beam can only penetrate into the target up to a point, where the plasma frequency $\omega_{\mathrm{p}}$ equals the frequency of the wave $\omega_{\mathrm{L}}$. The corresponding density at this point is called critical density ncrit and can be calculated $\left(\omega_{\mathrm{p}}=\omega_{\mathrm{L}}\right)$ from equation (3) [9]:

$$
n_{c r i t}=\frac{\omega_{L}^{2} \varepsilon_{0} m_{e}}{e^{2}}
$$

or, in terms of the wavelength (in microns),

$$
n_{\text {crit }}=\frac{1.11 \times 10^{21} \mathrm{e} / \mathrm{cm}^{3}}{\lambda^{2}(\mu \mathrm{m})}
$$

At the critical density many complex processes occur, but in a simple harmonic oscillation picture, the plasma-electrons are excited at their resonance frequency and absorb a large part of the electromagnetic radiation. Although this picture is strongly simplified, absorption at the so-called critical surface (where $n_{e}=$ $\mathrm{n}_{\text {crit }}$ ) is very important for the energy transfer between electromagnetic wave and the plasma. It should be noted that $\mathrm{n}_{\text {crit }}$ basically depends on the wavelength of the electromagnetic wave.

\section{Stimulated Electromagnetic Emissions}

The simulation part has been designed to simulate interaction of EM with ionosphere. Based on their different heights above the Earth surface, where atmospheric layers have been known with their designations as $\mathrm{D}-\mathrm{E}-\mathrm{F}$ layers. In the context of the present work, the thickness of each layer is considered as one of the input data parameters which are essentially required as initial conditions of the software measures. these layers have been playing an important role in many applications such as weather, jet streams, Earth protection from falling objects, and also radio and satellite communication and broadcasting, it is essential to study these. In table (3-1), 
some characteristic parameters of these layers are listed which are consider in the data processing carried out by the COMSOL physics presented in this paper.

Selection of these parameters is justified by the fact that the ionosphere layer is containing a high density of free electron and ions which are susceptible for energy -energy coupling with electromagnetic waves passing through this layer. if not otherwise mentioned, affixed wave frequency such $2.45 \mathrm{GHz}$ was considered when a master of this frequency and a certain power interact with the free electron in the ionosphere region. such layer can be optically considered as a mirror for these interacting electromagnetic waves as the electron density may vary along in a particular system of coordinates. the input data include wave frequency electron density and layer thickness. the output of the program depicts the impact of the wave power on the electron temperature, plasma potential], electric field norm and resistive losses, the frequency values $(2.45 \mathrm{GHz}, 1 \mathrm{GHz}$ and $10 \mathrm{MHz})$. With the frequency $1 \mathrm{G}$ the values of electron density $\left(10^{8}-10^{9)}\right.$ take very long time without given or complete the simulation so that this values don't have the result of simulation.

Table (3-1) characteristics parameter of ionosphere layers

\begin{tabular}{|c|c|c|c|}
\hline \multicolumn{2}{|c|}{$\begin{array}{c}\text { IONOSPHER } \\
\text { LAYERS }\end{array}$} & $\begin{array}{c}\text { ELECTRON } \\
\text { DENSITY } \mathrm{m}^{-3}\end{array}$ & Nominal Thickness km \\
\hline \multirow{2}{*}{$\mathrm{D}$} & $\mathrm{D} 1$ & $10^{8}$ & 30 \\
\cline { 2 - 4 } & $\mathrm{D} 2$ & $10^{9}$ & 30 \\
\hline \multirow{2}{*}{$\mathrm{E}$} & $\mathrm{E} 1$ & $10^{10}$ & 60 \\
\cline { 2 - 4 } & $\mathrm{E} 2$ & $10^{11}$ & 60 \\
\hline \multirow{2}{*}{$\mathrm{F}$} & $\mathrm{F} 1$ & $10^{11}$ & 350 \\
\cline { 2 - 4 } & $\mathrm{F} 2$ & $10^{12}$ & 350 \\
\hline
\end{tabular}

The simulation has been designed by using COMSOL multiphysics program, in which 3 Ionosphere layers (D, E, F) has been presented for simulation model to steady the effect of electromagnetic wave frequency on the electron density, and electron temperature as shown in figure 3.

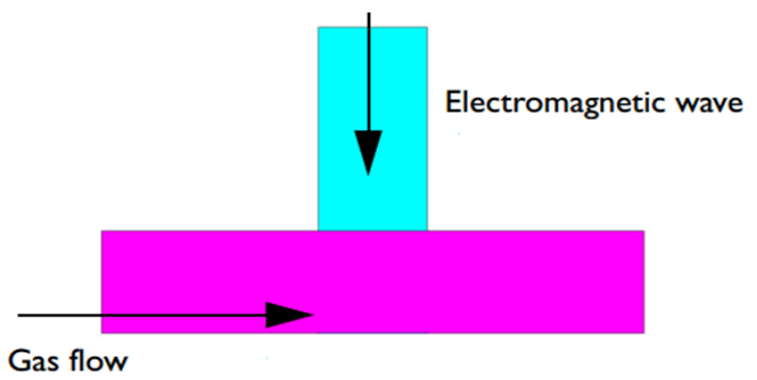

Figure (3): Diagram of geometry modeled. The electromagnetic wave enters from the top port and intersects the gas flow leading to the formation of a plasma.

As theory the ionized region can affect the transmission of radio waves in at least two ways. Firstly, under suitable conditions, charged particles (electrons) can remove energy from an electromagnetic wave and thus attenuate the signal; in the limiting case, the energy of the wave can be absorbed completely. Second, a wave travelling from one place to another in which the electron density is different will undergo a change in its direction of propagation, due to the effect of free electrons on the velocity of a radio wave. These two effects of an ionized medium on electromagnetic radio waves are explained here in our model.

\section{Simulation Results}

The electron densities were selected for each layer where these electrons were allowed to interact with a wave of two frequencies one of which is the corresponding plasma frequency for the electron density. The energy coupling in the interaction is efficient or not, the orange color for example efficient interaction plasmawave, while the blue color illustrates a weak level of such interaction. It is worth mentioning that result obtained for each wave frequency will provide 6 plots demonstrating the characteristic behavior of a particular parameter after the interaction, this characteristic parameter is electron density, electron temperature, electric potential electric, field norm resistive losses and power input (power time relationship). Results also cover the doming mode of the interaction which is determine by the amount of energy responsible for exciting such mode. transverse electric and transverse magnetic mode with different order were obtained as inherently figured out by the program simulation. For our test, we have been simulated the interaction of the radio waves of frequencies $(2.45 \mathrm{GHz}$ and $10 \mathrm{MHz})$ with ionosphere $\mathrm{D}, \mathrm{E}$ and $\mathrm{F}$ layers. 


\subsection{The D- layer}

This layer is located at height of 50-80 km above the Earth surface which may contain, as other layers, free electrons and ions generated by solar radiation.

\subsubsection{The Simulation results for interaction of electromagnetic waves of $2.45 \mathrm{GHz}$ frequency with D-layer of $\left(10^{8} \mathrm{~m}^{-3}\right)$ electron density, are shown in figure 4 .}

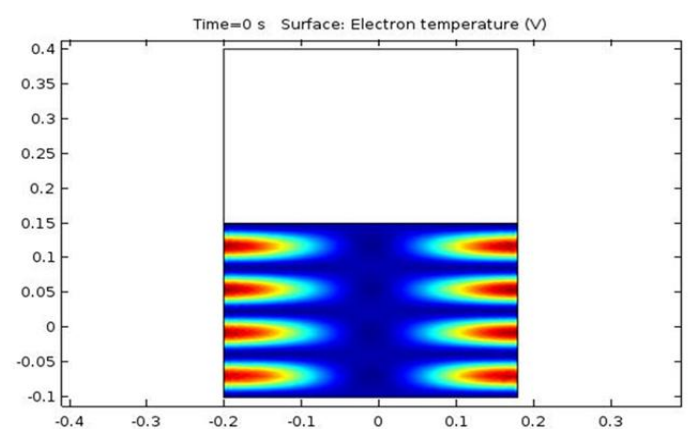

(a)

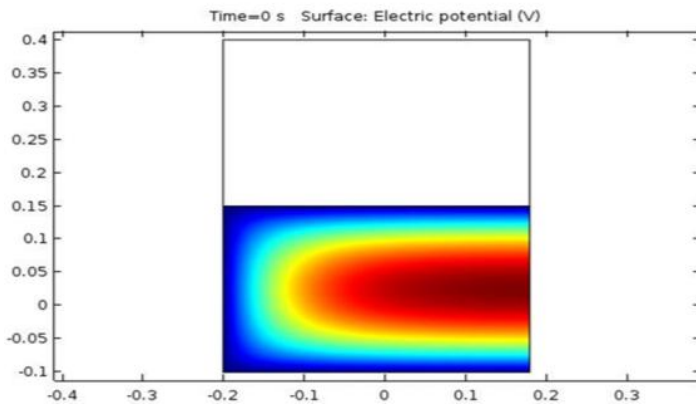

(c)

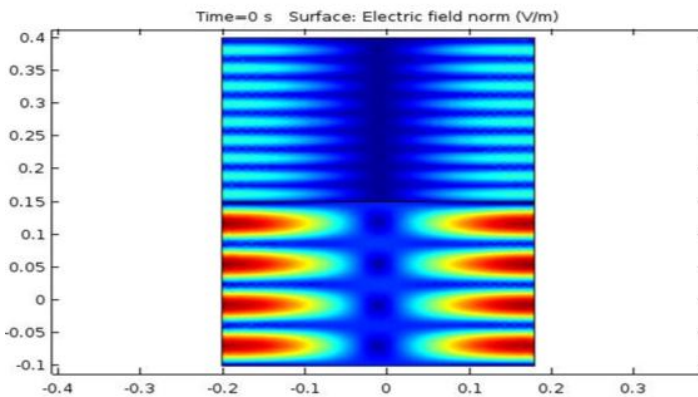

(e)

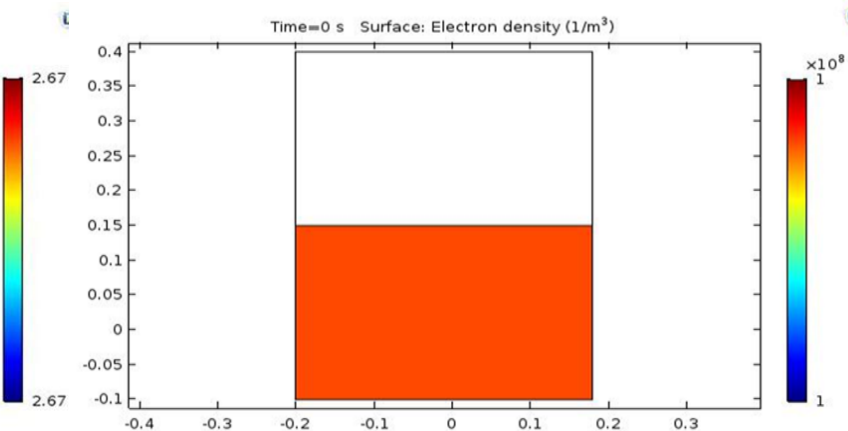

(b)

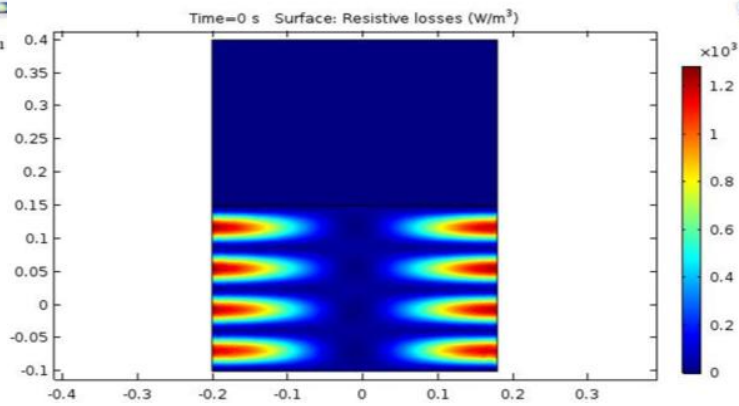

(d)

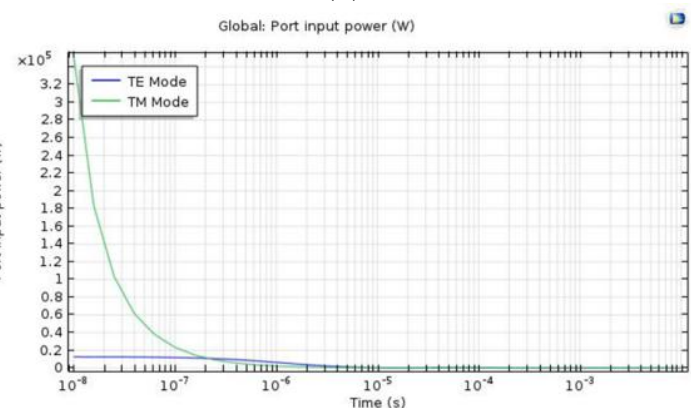

(f)

Figure (4): Results Of Interaction Of The Wave Frequency 2.45ghz With D-Layer, (A) Electron Temperature Of Plasma, (B) Electron Density Of Plasma, (C) Electric Potiential Of Plasms, (D) Resistive Losses, (E) Electric Field Norm, (F) Curve That Shown The Relation Between The Input Port Power And The Time

The electron "temperature" is plotted in Figure 3(a). The electron temperature is relatively low everywhere except some places in the way of the wave inter the plasma can be seen in Figure 3(b) the electron density. the electric potential plot in figure 4(c) its value in the regions where the interaction is efficient is $7 * 10^{-11}$ volt. in figure 4 (d) we show the resistive losses the higher value in the efficient region is $1.2 * 10^{3} \mathrm{w} / \mathrm{m} 3$. The electric field is high inside the waveguide and there are no losses. Once the wave is exposed to the plasma, the energy is absorbed by the electrons, raising the electron temperature enough to generate new electrons through ionization. The ionization rate is high enough to sustain the plasma. The electromagnetic wave cannot penetrate into regions exceeding the critical plasma density. Since the electron "temperature" is relatively low, one would expect the plasma potential to be low that we show in figure 4(e). In the figure 4(f) we show that the Tm mode is domned mode, electrons do not experience any change in the high-frequency electric field during the microwave time scale. This means that the phase coherence between the electrons and electromagnetic waves is only destroyed through collisions with the background gas. The loss of phase coherence between the electrons and high-frequency fields is what results in energy gain for the electrons 


\subsubsection{The Simulation results for interaction of electromagnetic waves of $10 \mathrm{MHz}$ frequency with D-layer} of $\left(10^{8} \mathrm{~m}^{-3}\right)$ electron density, are shown in figure 5 .

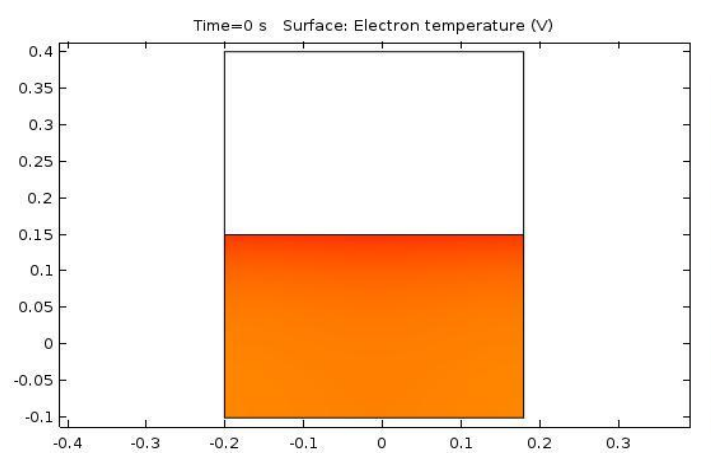

(a)

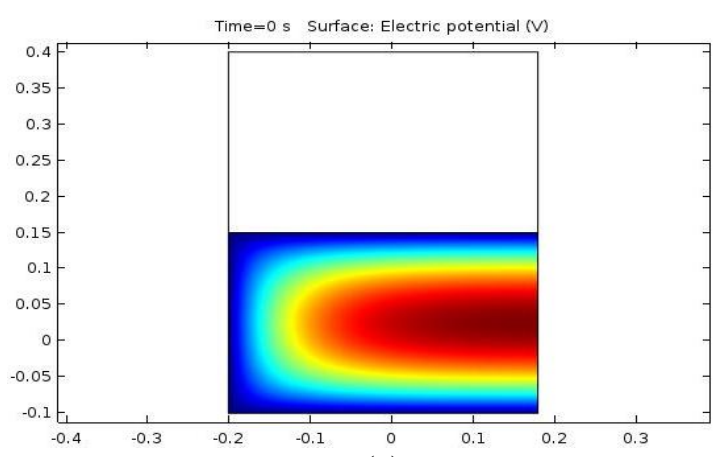

(c)

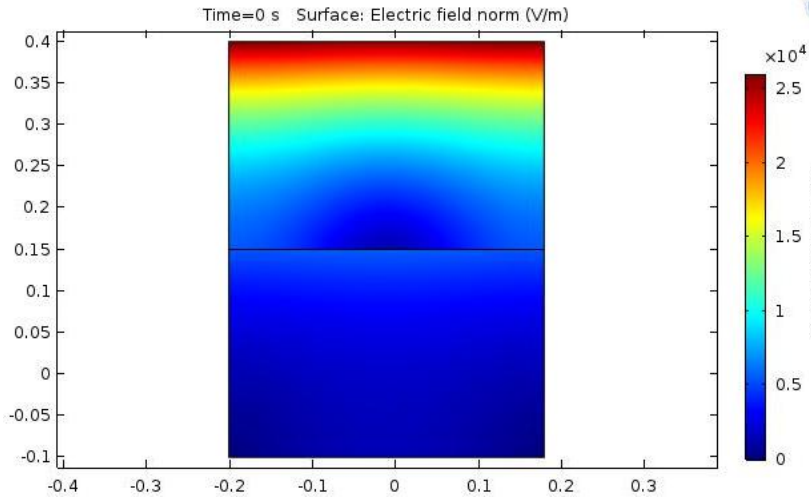

(e)

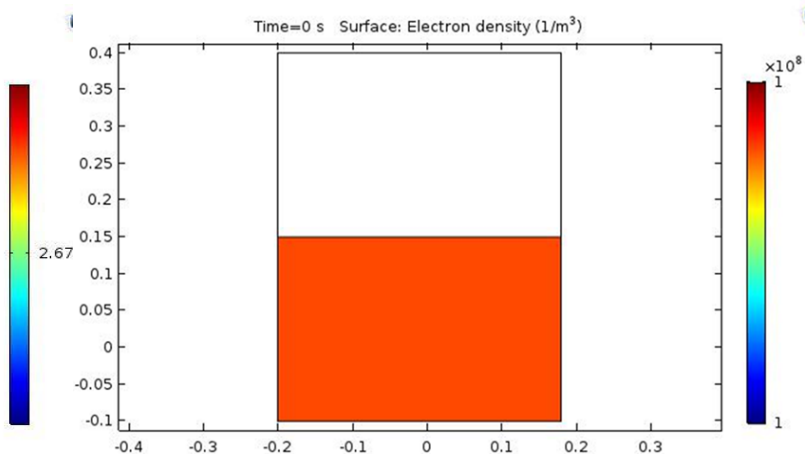

(b)

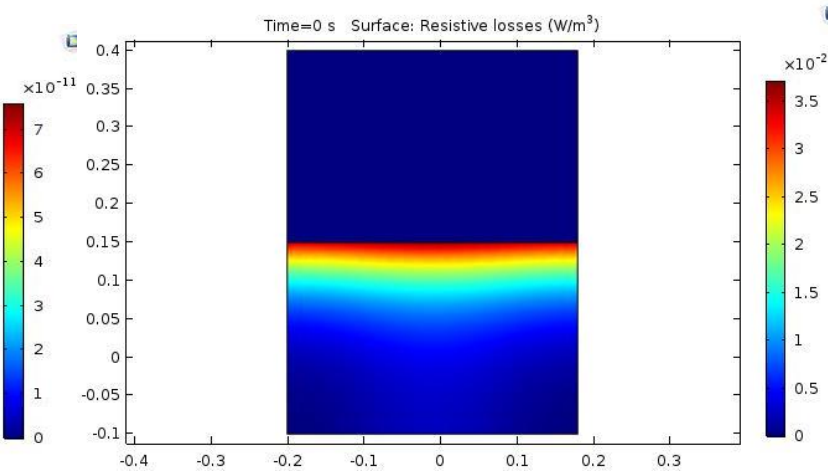

(d)

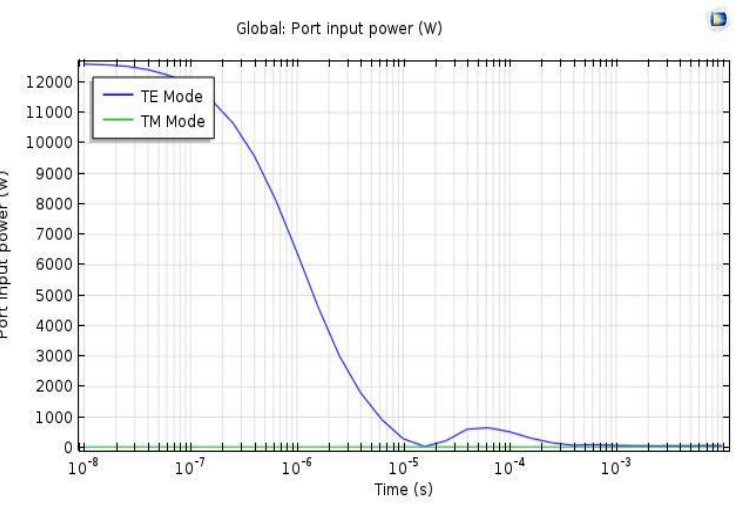

(f)

Figure (5): results of interaction of the wave frequency $10 \mathrm{MHz}$ with D-layer, (a) electron temperature of plasma, (b) electron density of plasma, (c) electric potiential of plasms, (d) resistive losses, (e) electric field norm, (f) curve that shown the relation between the input port power and the time.

The electron "temperature" is plotted in Figure 5(a). The electron temperature is relatively high everywhere can be seen in Figure 5(b) the electron density. the electric potential plot in figure 5(c) its value in the regions where the interaction is efficient is $7 * 10^{-11}$ vote. in figure $5(\mathrm{~d})$ we show the resistive losses the higher value in the efficient region is $1.2 * 10^{-2} \mathrm{w} / \mathrm{m} 3$. The electric field is high inside the waveguide and there are no losses. Once the wave is exposed to the plasma, the energy is absorbed by the electrons, raising the electron temperature enough to generate new electrons through ionization. The ionization rate is high enough to sustain the plasma. The electromagnetic wave cannot penetrate into regions exceeding the critical plasma density. Since the electron "temperature" is relatively low, one would expect the plasma potential to be low that we show in figure 5(e). In the figure 5(f) we show that the TE mode is domned mode.

\subsection{The E- layer}

This layer is located at height of $80-110 \mathrm{~km}$ above the Earth surface which may contain, as other layers, free electrons and ions generated by solar radiation. 
6.2.1 The Simulation results for interaction of electromagnetic waves of frequency $2.45 \mathrm{GHz}$ with E-layer of $10^{10} \mathrm{~m}^{-3}$ electron density, are shown in figure 6 .

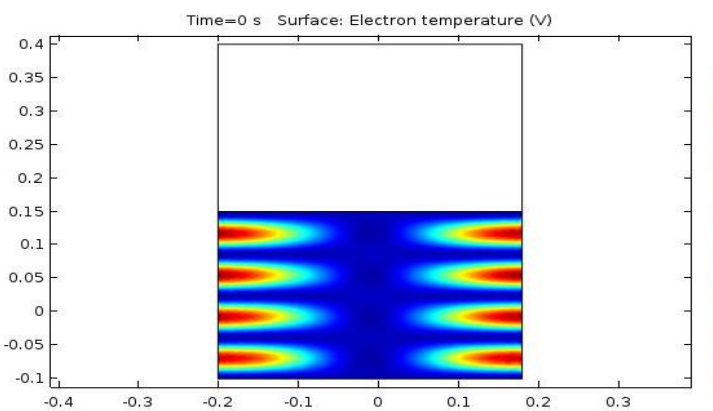

(b)

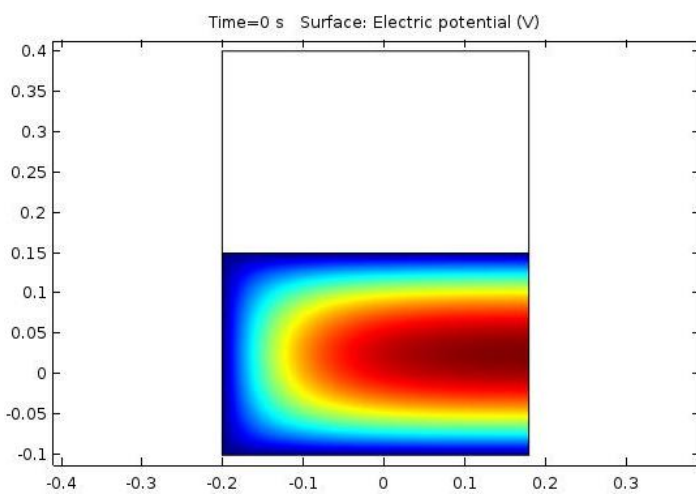

(c)

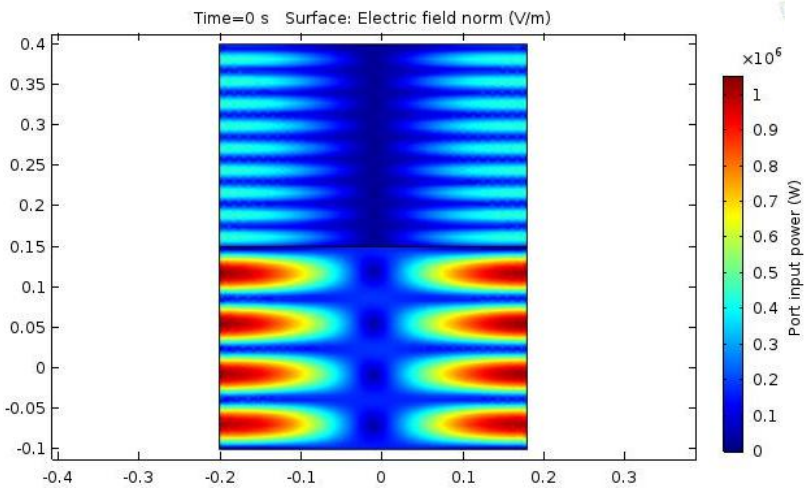

(e)

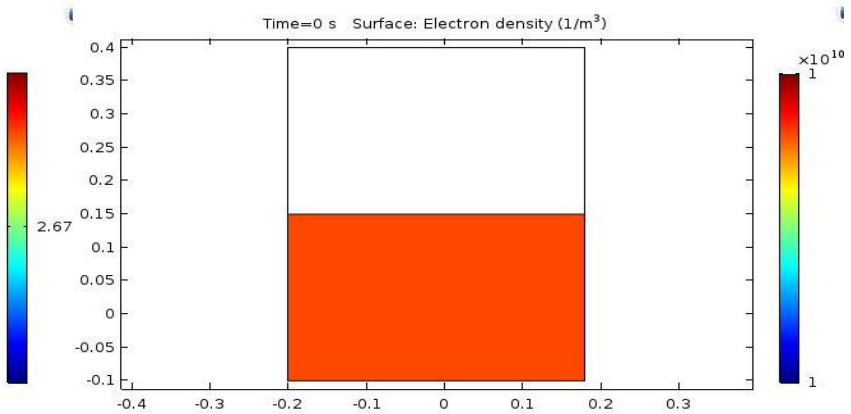

(b)

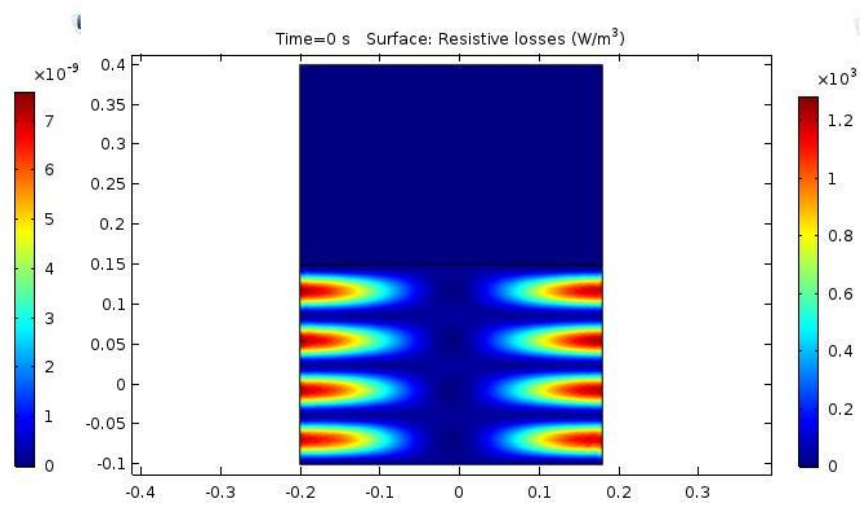

(d)

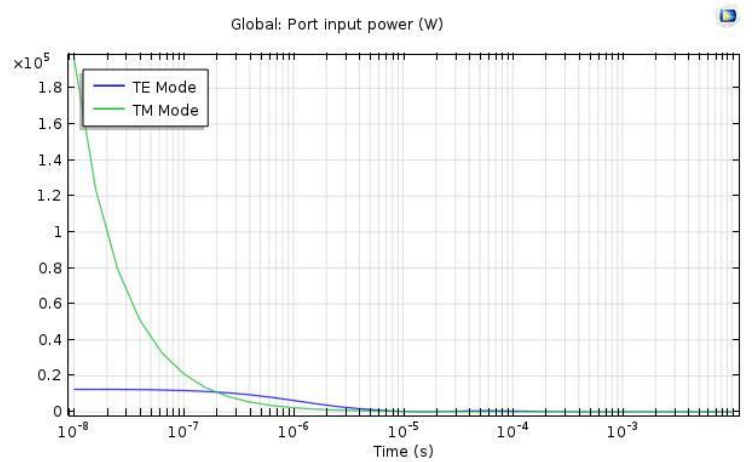

(f)

Figure (6): results of interaction of the wave frequency $2.45 \mathrm{GHz}$ with E-layer, (a) electron temperature of plasma, (b) electron density of plasma, (c) electric potiential of plasms, (d) resistive losses, (e) electric field norm, (f) curve that shown the relation between the input port power and the time

The electron "temperature" is plotted in Figure 6(a). The electron temperature is relatively low everywhere except some region in the way of wave, can be seen in Figure 6(b) the electron density. The electric potential plote in figure $6(\mathrm{c})$ its value in the regions where the interaction is efficient is $7 * 10^{-9}$ volt. In figure 6(d) we show the resistive losses the higher value in the efficient region is $1.2 * 10^{3} \mathrm{w} / \mathrm{m} 3$. The electric field is high inside the waveguide and there are no losses. Once the wave is exposed to the plasma, the energy is absorbed by the electrons, raising the electron temperature enough to generate new electrons through ionization. The ionization rate is high enough to sustain the plasma. The electromagnetic wave cannot penetrate into regions exceeding the critical plasma density. In the figure 6(f) we notice that the TM mode is dominant. 


\subsubsection{Simulation results for interaction of electromagnetic waves of frequency $10 \mathrm{MHz}$ with E-layer with} electron density $10^{10} \mathrm{~m}^{-3}$ shown in figure 7.

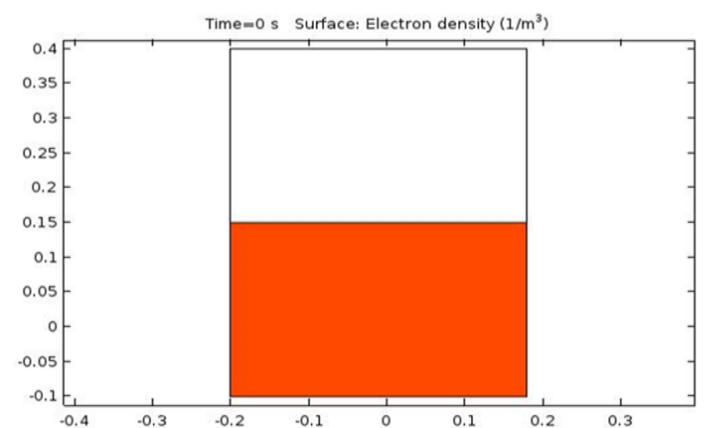

(c)

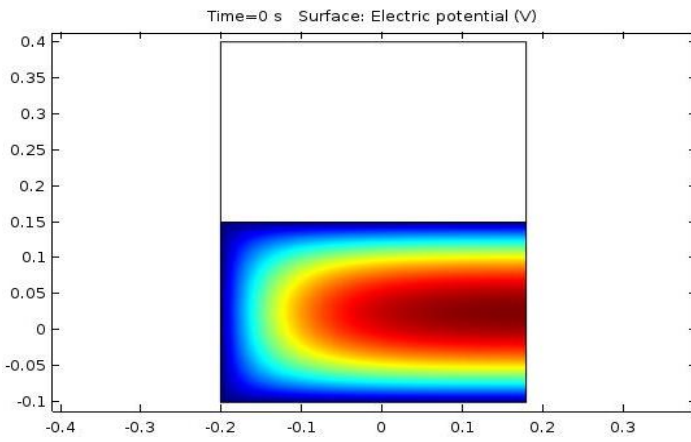

(c)

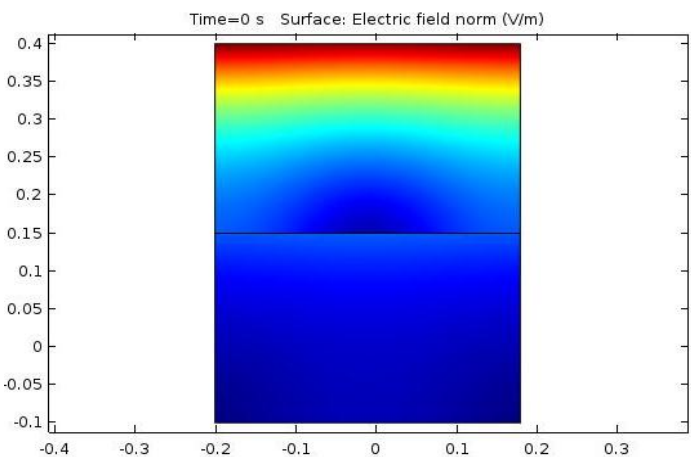

(e)

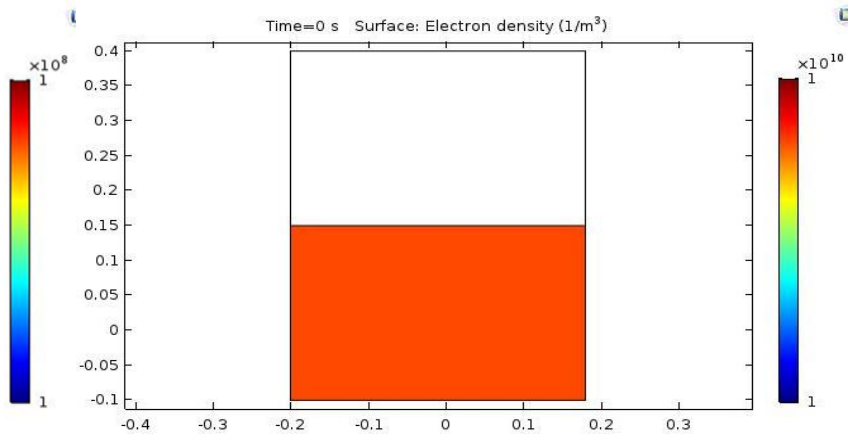

(b)

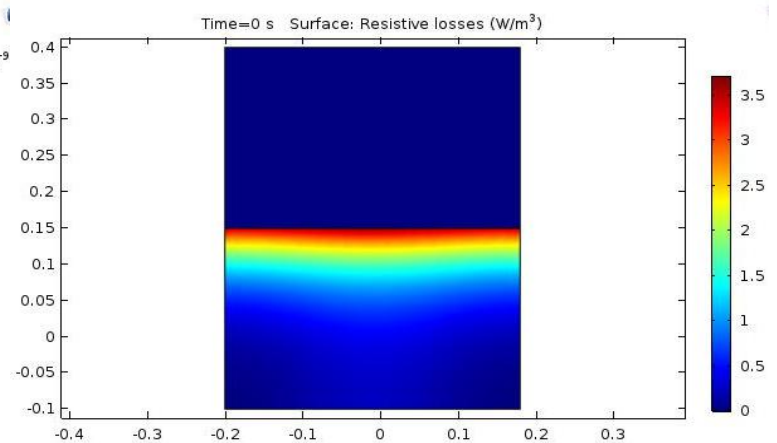

(d)

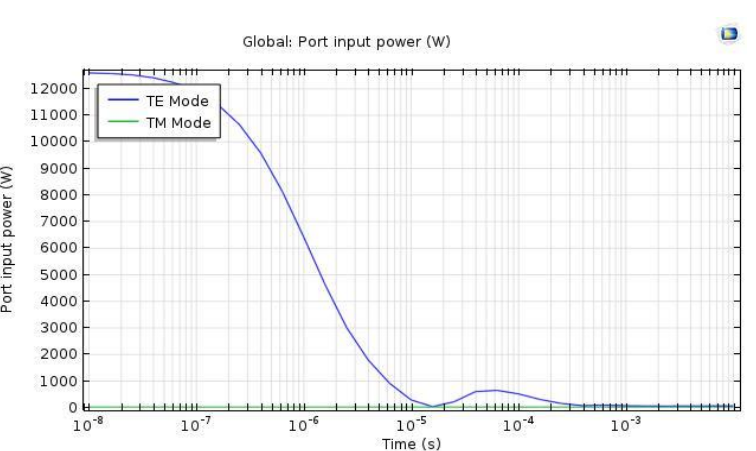

(f)

Figure (7): results of interaction of the wave frequency $10 \mathrm{MHz}$ with E-layer, (a) electron temperature of plasma, (b) electron density of plasma, (c) electric potential of plasms, (d) resistive losses, (e) electric field norm, (f) curve that shown the relation between the input port power and the time

The electron "temperature" is plotted in Figure 7(a). The electron temperature is relatively HIGH everywhere, can be seen in Figure 7(b) the electron density. the electric potential plot in figure 7(c) its value in the regions where the interaction is efficient is $7 * 10^{-9}$ volt. In figure $7(\mathrm{~d})$ we show the resistive losses the higher value in the efficient region is $3.5 \mathrm{w} / \mathrm{m} 3$. In figure 7 (e) show the electric field norm is high inside the waveguide and there are no losses. Once the wave is exposed to the plasma, the energy is absorbed by the electrons, raising the electron temperature enough to generate new electrons through ionization. The ionization rate is high enough to sustain the plasma. The electromagnetic wave cannot penetrate into regions exceeding the critical plasma density. In the figure 7(f) we notice that the TE mode is dominant.

\subsection{The F- layer}

This layer is located at height of $110-550 \mathrm{~km}$ above the Earth surface which may contain, as other layers, free electrons and ions generated by solar radiation. this layer usually shows its affecting observations during night time when there is no solar radiation interacting with electron and ions. 
6.2.1 The Simulation results for interaction of electromagnetic waves of frequency $2.45 \mathrm{GHz}$ with F-layer of $10^{11} \mathrm{~m}^{-3}$ electron density, are shown in figure 8 .

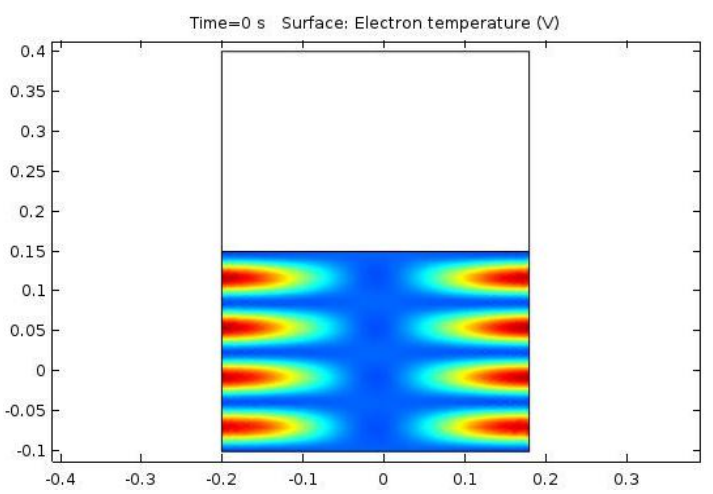

(d)

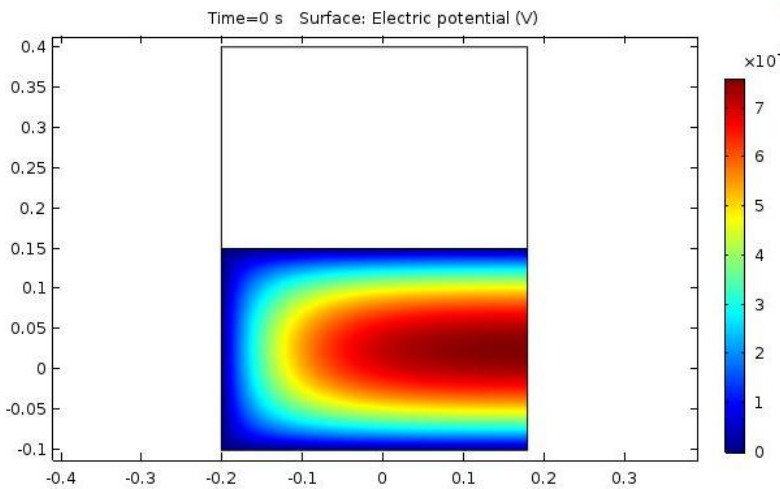

(c)

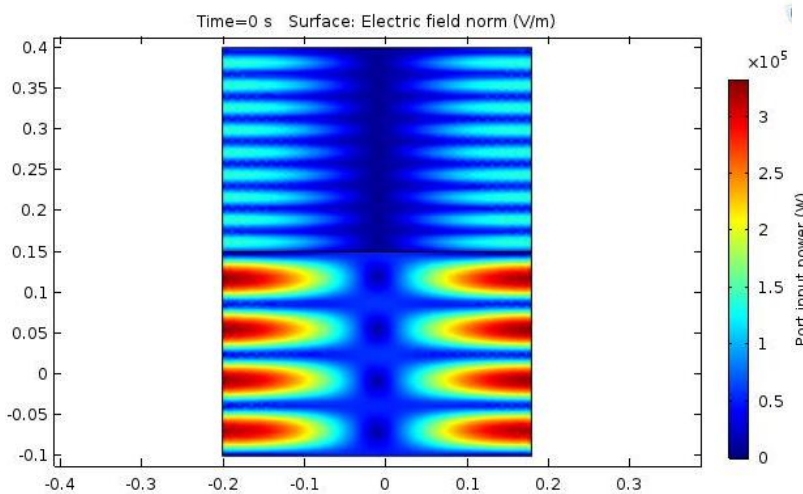

(e)

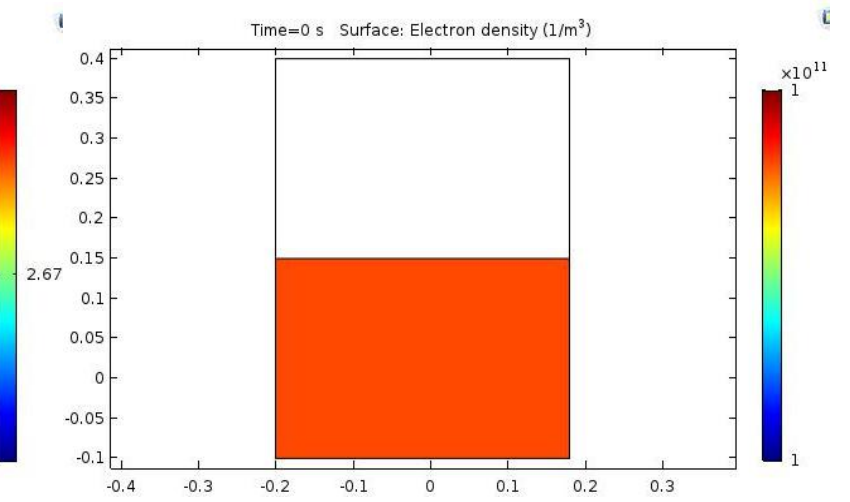

(b)

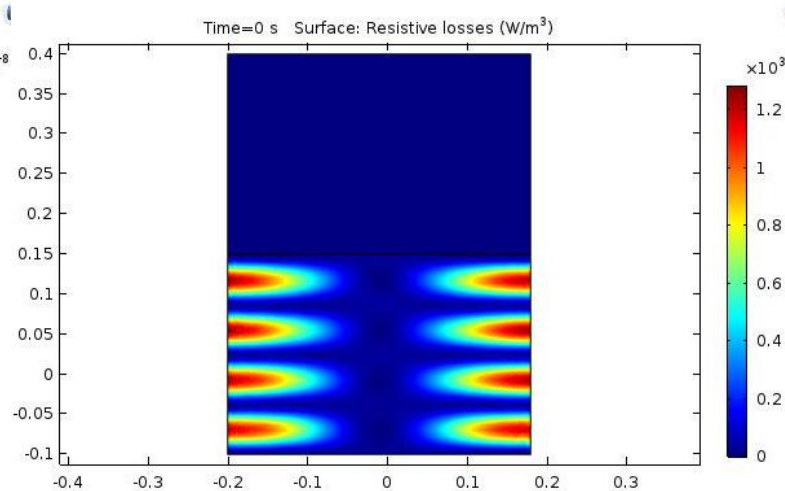

(d)

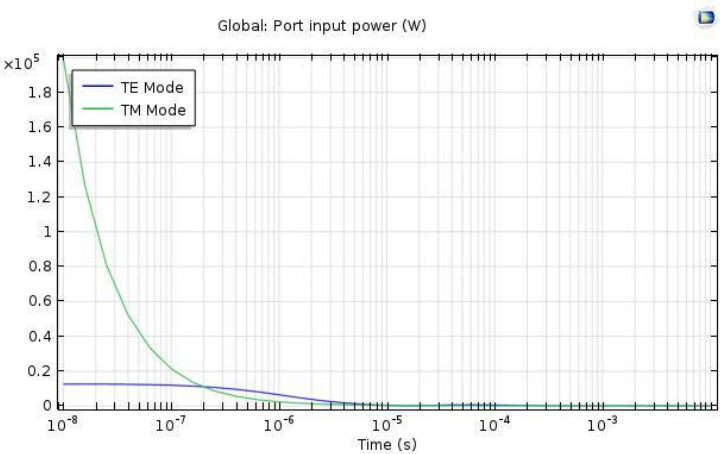

(f)

Figure (8): results of interaction of the wave frequency $2.45 \mathrm{GHz}$ with F-layer, (a) electron temperature of plasma, (b) electron density of plasma, (c) electric potential of plasms, (d) resistive losses, (e) electric field norm, (f) curve that shown the relation between the input port power and the time

The electron "temperature" is plotted in Figure 8(a). The electron temperature is relatively low everywhere expect some region in way of the wave, can be seen in Figure 8(b)the electron density Show that the interaction is efficient everywhere. the electric potential plot in figure 8(c) its value in the regions where the interaction is efficient is $7 * 10^{-8}$ volt. In figure 8 (d) we show the resistive losses the higher value in the efficient region is $10^{3} \mathrm{w} / \mathrm{m} 3$. In figure $8(\mathrm{e})$ show the electric field norm is high inside the waveguide and there are no losses. Once the wave is exposed to the plasma, the energy is absorbed by the electrons, raising the electron temperature enough to generate new electrons through ionization. The ionization rate is high enough to sustain the plasma. The electromagnetic wave cannot penetrate into regions exceeding the critical plasma density. In the figure 8(f) we notice that the TM mode is dominant. 
6.3.2 Simulation results for interaction of electromagnetic waves of frequency $10 \mathrm{MHz}$ with F-layer with electron density $10^{11} \mathrm{~m}^{-3}$ shown in figure 9.

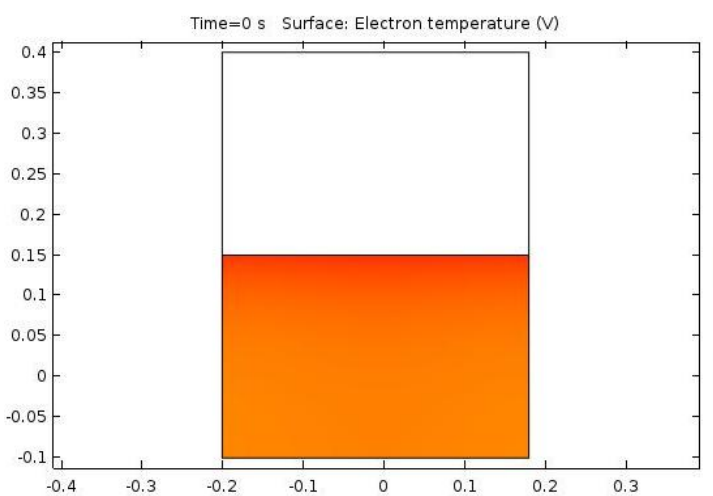

(e)

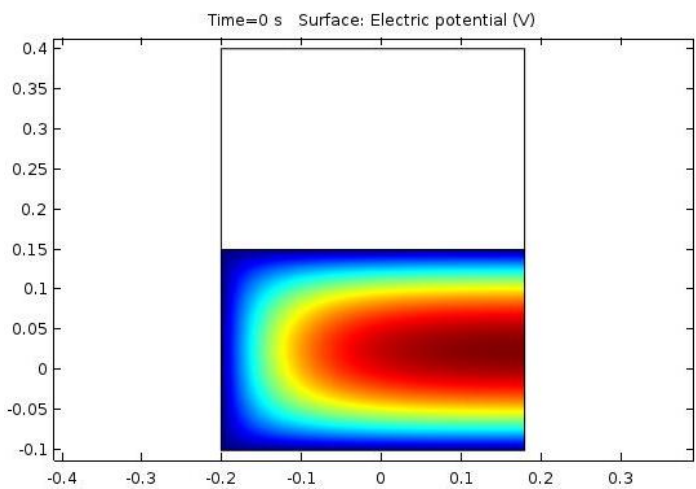

(c)

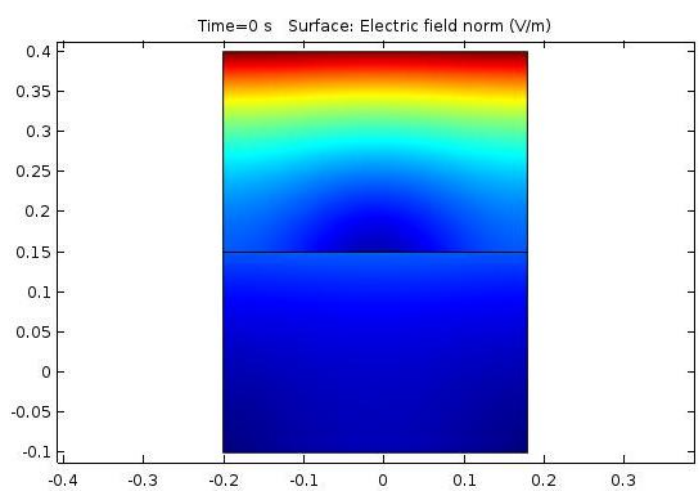

(e)

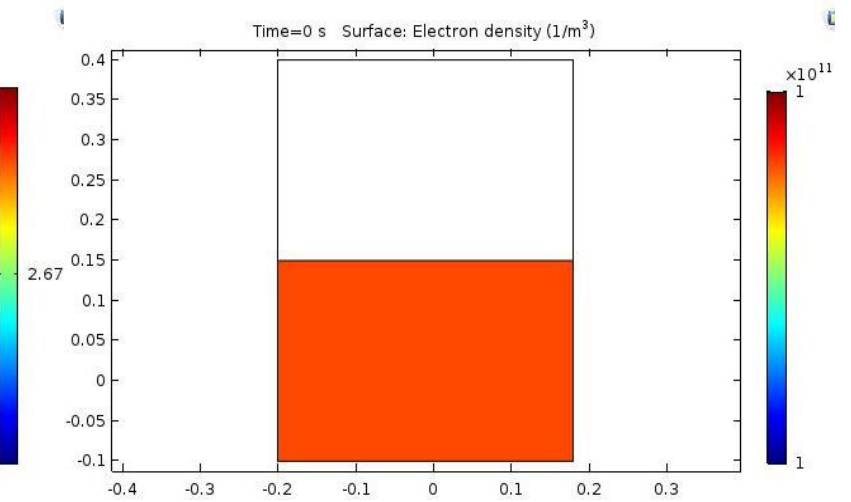

(b)

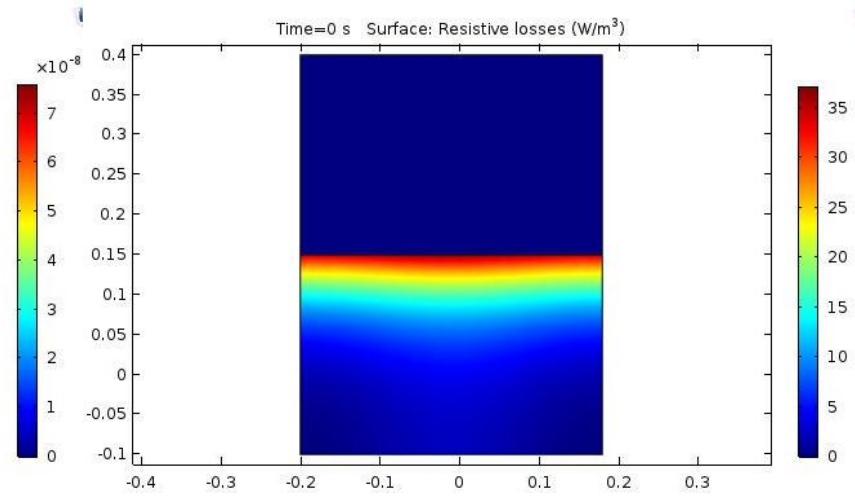

(d)

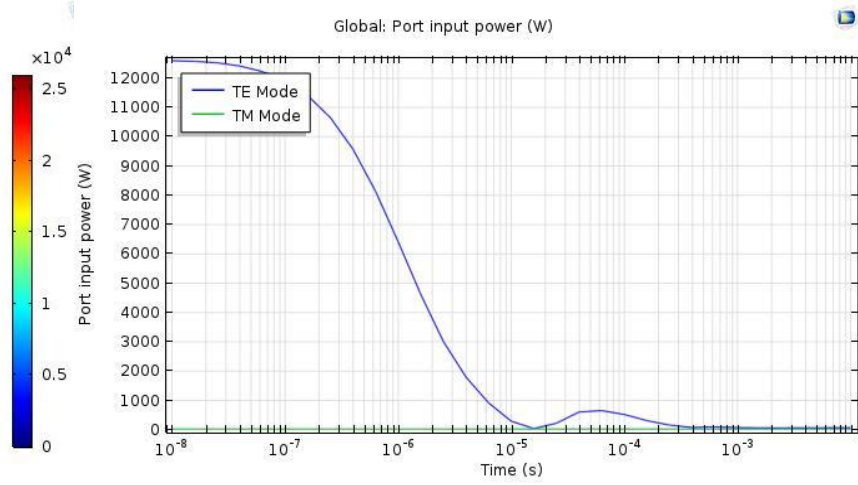

(f)

Figure (9): results of interaction of the wave frequency $10 \mathrm{MHz}$ with F-layer, (a) electron temperature of plasma, (b) electron density of plasma, (c) electric potential of plasms, (d) resistive losses, (e) electric field norm, (f) curve that shown the relation between the input port power and the time

The electron "temperature" is plotted in Figure 9(a). The electron temperature is relatively high everywhere, can be seen in Figure 9(b) the electron density. The electric potential plot in figure 9(c) its value in the regions where the interaction is efficient is $7 * 10^{-8}$ volt. in figure $9(\mathrm{~d})$ we show the resistive losses the higher value in the efficient region is $2.4 * 10^{4} \mathrm{w} / \mathrm{m}^{3}$. In figure 9(e) show the electric field norm is high inside the waveguide and there are no losses. Once the wave is exposed to the plasma, the energy is absorbed by the electrons, raising the electron temperature enough to generate new electrons through ionization. The ionization rate is high enough to sustain the plasma. The electromagnetic wave cannot penetrate into regions exceeding the critical plasma density. In the figure 9(f) we notice that the TE mode is dominant.

\section{Conclusion}

From the simulation Results that simulate interaction of EM waves we noticed that the wave frequency of $2.45 \mathrm{GHz}$ and the frequency equal to the plasma frequency has shown various level of interaction demonstrated by parameter under considerate including electron density, electron temperature, electric potential, 
electric field norm and resistive losses. In addition, the electron temperature is relatively high everywhere, in part due to the low operating pressure (mbar). For electric field results, it shown that the electric field is high inside the waveguide and there are no losses. Once the wave is exposed to the plasma, the energy is absorbed by the electrons, raising the electron temperature enough to generate new electrons through ionization. For ionization rate, the results show it is high enough to sustain the plasma. In the TE mode, electrons do not experience any change in the high-frequency electric field during the microwave time scale. This means that the phase coherence between the electrons and electromagnetic waves is only destroyed through collisions with the background gas. The results are approved that the loss of phase coherence between the electrons and highfrequency fields is what results in energy gain for the electrons. Also, the electric field cannot penetrate past the contour of critical electron density, and has its greatest magnitude in this location, and the TM mode causes inplane motion of the electrons on the microwave time scale, so in regions where the high-frequency electric field is significant (the contour where the electron density is equal to the critical density).

\section{References}

[1] J TH Pau, "Electromagnetic Wave Interaction with the Auroral Plasma", PhD Thesis, Physics, University of California, Los Angeles, 2003

[2] A Yeşil, S Sağır and O Özcan, "Comparison of Maximum Electron Density Predicted by Iri-2001 With That Measured Over Chilton Station", e-Journal of New World Sciences Academy, Physical Sciences, 3A0011, 4, (3), 92-98, 2009.

[3] D L. Tang, A P Sun, X M Qiu, and Paul K Chu, "Interaction of Electromagnetic Waves with a Magnetized Nonuniform Plasma Slab", IEEE Transactions On Plasma Science, VOL. 31, NO. 3, 2003.

[4] C S Gurel and E Oncu, "Interaction of Electromagnetic Wave and Plasma Slab with Partially Linear and Sinusoidal Electron Density Profile", Progress In Electromagnetics Research Letters, Vol. 12, 171-181, 2009.

[5] K Papadopoulos, C L Chang, J Labenski, and T Wallace, " The first demonstration of HF-driven ionosphere currents", Geophysical Research Letters, VOL. 38, 2011

[6] A Yeşil and İ Ünal, "Electromagnetic Wave Propagation in Ionospheric Plasma”, InTech Europe, Vol 10, pp.190-212, 2011.

[7] K Mursula and T Ulich, "A new method to determine the solar cycle length", Geophysics Research Letters, vol. 25,1873$1840,1998$.

[8] T B Leyser,and AY Wong, "Powerful electromagnetic waves for active environmental research in geospace", American Geophysical Union, Reviews of Geophysics, 47, RG1001, 1 of 33, doi:10.1029/2007RG000235, 2009.

[9] Herbert C. Carison, Jr, "High Power HF Modification: Geophysics, Span of EM Effects, And Energy Budget”, Adv. Space Res. Vol. 13, No. 10, pp. (10)15 - (10)24, 1993 